\title{
Mantel-Haenszel Test Statistics for Correlated Binary Data
}

\author{
by \\ Jie Zhang and Dennis D. Boos \\ Department of Statistics, North Carolina State University \\ Raleigh, NC 27695-8203 \\ tel: (919) 515-1918 \\ fax: (919) 515-7591 \\ e-mail: jzhang@stat.ncsu.edu, boos@stat.ncsu.edu
}

March, 1996 


\section{Mantel-Haenszel Test Statistics for Correlated Binary Data}

\section{Summary}

This paper proposes two new Mantel-Haenszel test statistics for correlated binary data in $2 \times 2$ tables that are asymptotically valid in both sparse data (many strata) and largestrata limiting models. Monte Carlo experiments show that the statistics compare favorably to previously proposed test statistics, especially for 5-25 small to moderate-sized strata. Confidence intervals are also obtained and compared to those obtained from the test of Liang (1985). 


\section{Introduction}

Multicenter randomized clinical trials are frequently used to test the efficacy of new medical regimens. When the response variables are binary, and there are only two treatment groups (drug and placebo), the data often can be summarized in a series of $2 \times 2$ tables based on some covariates or strata such as the clinical centers. We will illustrate this by the following two examples.

\section{Example 1. A psoriasis multicenter clinical trial}

The first example is from a clinical trial comparing a new drug and a placebo for treatment of psoriasis. In each of the sixteen centers, patients were randomly assigned to one of the two treatments and were evaluated on a $0-1$ scale $(0=$ no improvement, $1=$ improvement $)$ at four following visits. The summarized data are in Table A1 in the Appendix. The full data for 11 of the 277 patients (from Center 2) are in Table A2.

\section{Example 2. A heartburn multicenter clinical trial}

At the beginning of a multicenter clinical trial for testing the efficacy of a new drug for heartburn relief, patients were randomly assigned to drug or placebo in each of the seventeen clinical centers. For the next two weeks these patients took the given medication by self-administration at each onset of heartburn symptoms, and then recorded the medication results in their diary cards. If the medication succeeded in relieving the heartburn symptoms, a "1" (success) was recorded, otherwise, a "0" (failure) was recorded. The data structure is illustrated in Tables A3 and A4.

Both data sets have the following three characteristics.

1. The number of centers $(\mathrm{k})$ is moderately large, but the number of patients in many of the centers is small. This sparse data situation occurs for example when enrollment of large numbers of patients is not possible at each individual center. Furthermore, this sparse data situation will become more serious in the data analysis when adjustment for other prognostic factors is required. For example, we may want to adjust for patient smoking status when we analyze the data from the heartburn clinical trial.

2. The success rates for treatments vary appreciably from center to center. This is because patients at different centers may differ in some important respects that are difficult to measure. For instance, pollution levels, water hardness and other less tangible variables 
might have an effect on the responses. In addition, different nursing care and staff morale could have an effect on patients, too. Hence, adjustment for the center effect is important when we analyze these two data sets.

3. Dependence exists between observations due to repeated measurement on the same patient. This dependence may also be caused by subsampling from clusters such as family units in other situations. Such data are often called correlated or clustered binary data.

For simplicity, centers and strata based on cross-classification of centers and other prognostic factors will all be referred to as strata from now on. When the number of strata $(k)$ is small and the number of patients in each stratum is large, hypothesis tests may be based on the generalized estimating equations (GEE) approach of Liang and Zeger (1986), see also Rotnitzky and Jewell (1990) and Fitzmaurice et al. (1993), but standard GEE will not be appropriate in the sparse situation 1) above because of the many strata parameters that need to be estimated, see Liang and Zeger (1995) for further explanation. On the other hand, Mantel-Haenszel statistics and the conditional maximum likelihood approach are robust to the sparse situation 1) (Breslow, 1981), but the assumption of an underlying hypergeometric distribution is not satisfied under the correlated binary data as pointed out by Liang (1985). Hence, both of these methods will be invalid under the correlation situation 3 ) above.

Liang (1985) proposed two tests which handle the sparse correlated binary data, but their asymptotic validity depends on $(k) \rightarrow \infty$, which results in the requirement of a large number of strata $(k)$. More to the point, these tests are not very efficient since the variance estimates in the tests use strata as the primary sampling unit and are thus based on $k$ degrees of freedom. Donald and Donner (1987) also gave an adjusted Mantel-Haenszel test statistic for correlated binary data. Their statistic is based on estimating the intra-class correlation. This test statistic, however, requires an assumption of common correlation across the strata, and further, the estimator of intra-class correlation is not stable in the sparse case.

In this paper two new score tests for correlated binary data are proposed which require only the total number of patients to be reasonable large for asymptotic results to be applicable. Monte Carlo simulations compare these tests with the standard Mantel-Haenszel test, with one of Liang's (1985) tests, and with the test of Donald and Donner (1987). The simulations show 
that the new tests perform very favorably in situations with 5-25 strata and small to moderate strata sample sizes. Moreover the simulations confirm the validity of a simple power approximation which makes sample size calculations straightforward. Explicit confidence intervals for the common odds ratio are also derived from one of the new test statistics and from Liang's (1985) statistic. Monte Carlo comparisons are given for these intervals as well.

\section{Testing The Null Hypothesis of No Treatment Effect}

\subsection{Notation and Data Structure}

Let $n_{i j}$ and $m_{i j}$ represent the total repeated measurements on one patient or cluster size in the treatment and control groups, respectively, in stratum i, and further let $x_{i j}$ and $y_{i j}$ be the associated number of successes, where $\mathrm{i}=1,2, \ldots, k$. In addition, let $n_{i}$ and $m_{i}$ represent the number of patients in the treatment and control groups in stratum $\mathrm{i}$. Then $x_{i .}=\sum_{j=1}^{n_{i}} x_{i j}$ and $y_{i .}=\sum_{j=1}^{m_{i}} y_{i j}$ are the total number of successes in the ith stratum, and $n_{i .}=\sum_{j=1}^{n_{i}} n_{i j}$ and $m_{i .}=\sum_{j=1}^{m_{i}} m_{i j}$ are the total number of binary trials in the ith stratum. Hence, data from the ith strata) can be summarized in the following $2 \times 2$ table:

\begin{tabular}{lccc}
\multicolumn{4}{c}{ Responses for the ith stratum } \\
\hline \hline & success & failure & total \\
\hline treatment & $x_{i .}$ & $n_{i .}-x_{i .}$ & $n_{i .}$ \\
control & $y_{i .}$ & $m_{i .}-y_{i .}$ & $m_{i .}$ \\
\hline total & $t_{i .}$ & & $N_{i}$ \\
\hline \hline
\end{tabular}

We assume that all the patient results $x_{i j}$ and $y_{i j}$ are independent within and across the strata and that the expectations of $x_{i j}$ and $y_{i j}$ are equal to $n_{i j} p_{t i}$ and $m_{i j} p_{c i}$, where $p_{t i}$ and $p_{c i}$ are the treatment and control probabilities of success for a single binary trial in the ith stratum.

The common odds ratio $\Psi=\left\{p_{t i} /\left(1-p_{t i}\right)\right\} /\left\{p_{c i} /\left(1-p_{c i}\right)\right\}$ is the parameter of interest, and we will be testing $H_{0}: \Psi=1$. 


\subsection{Test Statistics}

The Mantel-Haenszel statistic

$$
T_{M H}=\frac{\left\{\sum_{i=1}^{k}\left(x_{i .}-n_{i .} t_{i .} / N_{i}\right)\right\}^{2}}{V_{M H}}
$$

has denominator

$$
V_{M H}=\sum_{i=1}^{k} \frac{n_{i .} m_{i .} t_{i .}\left(N_{i}-t_{i .}\right)}{N_{i}^{2}\left(N_{i}-1\right)}
$$

derived from the hypergeometric distribution. The related Cochran (1954) statistic based on the binomial distribution merely replaces $N_{i}-1$ by $N_{i}$ in the denominator of $V_{M H}$. In either case $V_{M H}$ is too small on average in the presence of positive within-cluster correlations.

Thus Liang (1985) proposed

$$
T_{L}=\frac{\left\{\sum_{i=1}^{k}\left(x_{i .}-n_{i .} t_{i .} / N_{i}\right)\right\}^{2}}{V_{L}}
$$

with variance estimate

$$
V_{L}=\sum_{i=1}^{k}\left(x_{i .}-n_{i .} t_{i .} / N_{i}\right)^{2} .
$$

Note that $T_{L}$ has the form of a squared t-statistic $\left(\sum Z_{i}\right)^{2} / \sum Z_{i}^{2}$ except that the null hypothesis assumption $E\left(Z_{i}\right)=0$ is used in the variance estimate instead of the sample average $\bar{Z}$. Under weak regularity conditions the central limit theorem yields an asymptotic chi-squared distribution with one degree of freedom for $T_{L}$ under $H_{0}$ as $k \rightarrow \infty$. If $k$ is fairly small, the chi-squared approximation will not be adequate, and in fact, it will be conservative. This can be seen by noting that if $Z_{1}, Z_{2}, \ldots$, and $Z_{k}$ are independent normal distributions with mean 0 and variance $\sigma^{2}$, then the mean and variance of $\left(\sum Z_{i}\right)^{2} / \sum Z_{i}^{2}$ are found to be 1 and $2-6 /(k+2)$, respectively. Similarly, the variance of $T_{L}$ under $H_{0}$ is often less than 2, resulting in conservative tests when a critical value from a chi-squared distribution with one degree of freedom is used. This also agrees with simulation results in Table 2.

Our two new test statistics have the same numerator as those of the Mantel-Haenszel test statistics above but different variance estimators in the denominator. The first one is 


$$
T_{P}=\frac{\left\{\sum_{i=1}^{k}\left(x_{i .}-n_{i .} t_{i .} / N_{i}\right)\right\}^{2}}{V_{P}},
$$

where

$$
V_{P}=\sum_{i=1}^{k}\left\{\left(1-\lambda_{i}\right)^{2} \sum_{j=1}^{n_{i}} \frac{\left(x_{i j}-n_{i j} \hat{p}_{i}\right)^{2}}{\left(1-n_{i j} / N_{i}\right)}+\lambda_{i}^{2} \sum_{j=1}^{m_{i}} \frac{\left(y_{i j}-m_{i j} \hat{p}_{i}\right)^{2}}{\left(1-m_{i j} / N_{i}\right)}\right\},
$$

with $\lambda_{i}=n_{i .} / N_{i}, \hat{p}_{i}=\left(x_{i .}+y_{i .}\right) / N_{i}$.

The motivation behind $V_{P}$ is as follows. To be consistent for correlated data, each of the stratum components of the variance estimate need to have the form of an empirical variance because we are not modeling the variance as a function of the mean. In addition, in the sparse data case where we are relying on laws of large numbers as $k \rightarrow \infty$, it is crucial that the ith component be approximately unbiased in order for the sum of variance estimates over strata to be consistent.

First note that the null expectation of the numerator of $T_{M H}$ is given by

$$
\sum_{i=1}^{k} \operatorname{Var}\left(x_{i .}-n_{i .} t_{i .} / N_{i}\right)=\sum_{i=1}^{k}\left\{\left(1-\lambda_{i}\right)^{2} \sum_{j=1}^{n_{i}} \operatorname{Var}\left(x_{i j}\right)+\lambda_{i}^{2} \sum_{j=1}^{m_{i}} \operatorname{Var}\left(y_{i j}\right)\right\} .
$$

If we knew the value of $p_{i}=p_{t i}=p_{c i}$ under $H_{0}$, then $\sum_{j=1}^{n_{i}}\left(x_{i j}-n_{i j} p_{i}\right)^{2}$ would be an unbiased estimate of $\sum_{j=1}^{n_{i}} \operatorname{Var}\left(x_{i j}\right)$. The variance estimate $V_{P}$ replaces $p_{i}$ by the pooled estimate $\hat{p}_{i}=$ $\left(x_{i .}+y_{i .}\right) / N_{i}$ and divides by $\left(1-n_{i j} / N_{i}\right)$ to adjust for this replacement. This adjustment works exactly when $\operatorname{Var}\left(x_{i j}\right)=n_{i j} c_{i}$ and $\operatorname{Var}\left(y_{i j}\right)=m_{i j} c_{i}$ for positive constants $c_{i}$. We summarize this result in the following theorem.

Theorem 1. If the $x_{i j}$ and $y_{i j}$ are all independent with means $E\left(x_{i j}\right)=n_{i j} p_{i}$ and $E\left(y_{i j}\right)=m_{i j} p_{i}$ and variances $\operatorname{Var}\left(x_{i j}\right)=n_{i j} c_{i}$ and $\operatorname{Var}\left(y_{i j}\right)=m_{i j} c_{i}$ for positive constants $c_{1}, \ldots, c_{k}$, then

$$
E\left(V_{P}\right)=E\left\{\sum_{i=1}^{k}\left(x_{i .}-n_{i .} t_{i .} / N_{i}\right)\right\}^{2} .
$$

Note that beta-binomial data does not satisfy the assumption of Theorem 1 that $\operatorname{Var}\left(x_{i j}\right)=n_{i j} c_{i}$ and $\operatorname{Var}\left(y_{i j}\right)=m_{i j} c_{i}$ except when $n_{i j}=m_{i j}$. Nevertheless, simulations for beta-binomial data in Table 2 show that $T_{P}$ works well even for unequal cluster sizes. 
Our second test statistic has denominator

$$
V_{U}=\sum_{i=1}^{k}\left\{\frac{\left(1-\lambda_{i}\right)^{2}}{\delta_{t i}} \sum_{j=1}^{n_{i}} \frac{\left(x_{i j}-n_{i j} \hat{p}_{t i}\right)^{2}}{\left(1-2 n_{i j} / n_{i .}\right)}+\frac{\lambda_{i}^{2}}{\delta_{c i}} \sum_{j=1}^{m_{i}} \frac{\left(y_{i j}-m_{i j} \hat{p}_{c i}\right)^{2}}{\left(1-2 m_{i j} / m_{i .}\right)}\right\},
$$

with $\hat{p}_{t i}=x_{i .} / n_{i .}, \hat{p}_{c i}=y_{i .} / m_{i .}$. and

$$
\delta_{t i}=1+\sum_{j=1}^{n_{i}} \frac{\left(n_{i j} / n_{i .}\right)^{2}}{\left(1-2 n_{i j} / n_{i .}\right)}, \quad \delta_{c i}=1+\sum_{j=1}^{m_{i}} \frac{\left(m_{i j} / m_{i .}\right)^{2}}{\left(1-2 m_{i j} / m_{i .}\right)} .
$$

The key difference between $V_{P}$ and $V_{U}$ is that $V_{P}$ uses the pooled estimator $\hat{p}_{i}$ whereas $V_{U}$ uses the unpooled estimators $\hat{p}_{t i}$ and $\hat{p}_{c i}$. $V_{U}$ seems more complicated than $V_{P}$, but it obtains the desired unbiasedness without any assumptions on the form of the variances of $x_{i j}$ and $y_{i j}$. The adjustments to achieve this unbiasedness follow easily upon noting that

$$
E\left(x_{i j}-n_{i j} \hat{p}_{t i}\right)^{2}=\operatorname{Var}\left(x_{i j}\right)\left(1-2 n_{i j} / n_{i .}\right)+\frac{n_{i j}{ }^{2}}{n_{i .}{ }^{2}} \sum_{j=1}^{n_{i}} \operatorname{Var}\left(x_{i j}\right) .
$$

Theorem 2. If the $x_{i j}$ and $y_{i j}$ are all independent with means $E\left(x_{i j}\right)=n_{i j} p_{t i}$ and $E\left(y_{i j}\right)=$ $m_{i j} p_{c i}$, then

$$
E\left(V_{U}\right)=E\left\{\sum_{i=1}^{k}\left(x_{i .}-n_{i .} t_{i .} / N_{i}\right)\right\}^{2} .
$$

Although $V_{U}$ is unbiased in general, we have found that $V_{P}$ is usually preferable because the pooled estimate $\hat{p}_{i}$ makes $V_{P}$ more stable than $V_{U}$ under $H_{0} . T_{P}$ has several additional properties as well. When $n_{i}=m_{i}=1$, i.e., in the case of matched pairs, $T_{P}$ reduces to Liang's $T_{L}$. In the case where $n_{i j}=m_{i j}=n_{0}$, the data can be placed in $k$ separate $2 \times\left(n_{0}+1\right)$ contingency tables. If SAS PROC FREQ is used with column scores equal to $0,1,2, \ldots, n_{0}$, and $n_{i}=m_{i}$, then the second Cochran-Mantel-Haenszel (CMH) statistic is exactly equal to $T_{P}$. This analysis might arise if one decided to analyze just the first $n_{0}$ episodes of each patient observed over time. This also shows that when $n_{0}=1$ (pure binary data), $T_{P}$ reduces to the usual Mantel-Haenszel statistic $T_{M H}$.

In a summary, both $T_{P}$ and $T_{U}$ have asymptotic chi-squared distributions when the total number of patients on treatment and control is large, that is, when $\sum_{i=1}^{k} n_{i} \rightarrow \infty$ and $\sum_{i=1}^{k} m_{i} \rightarrow$ $\infty$. Detailed summary of these asymptotic results is given in Theorems 3 and 4 in the Appendix, and proofs of these theorems are in Zhang and Boos (1995). 


\subsection{Power Calculations}

Following Wittes and Wallenstein $(1987,(2.3))$, the power of any of the Mantel-Haenszel tests may be approximated by

$$
\Phi\left(\frac{\sum_{i=1}^{k}\left(n_{i .} m_{i .} / N_{i}\right) \Delta_{i}}{\sqrt{V}}-Z(1-\alpha / 2)\right),
$$

where $\Phi$ is the standard normal distribution function, $\Delta_{i}=p_{t i}-p_{c i}$ is the difference of success probabilities for the ith center, $Z(1-\alpha / 2)$ is the $1-\alpha / 2$ quantile of a standard normal, and

$$
V=\sum_{i=1}^{k}\left\{\left(1-\lambda_{i}\right)^{2} \sum_{j=1}^{n_{i}} \operatorname{Var}\left(x_{i j}\right)+\lambda_{i}^{2} \sum_{j=1}^{m_{i}} \operatorname{Var}\left(y_{i j}\right)\right\}
$$

is the variance of the square root of the numerator of the Mantel-Haenszel statistics. For simplicity consider a study with the same alternative $\Delta_{i}=\Delta$, constant treatment and control probabilities $p_{t i}=p_{t}$ and $p_{c i}=p_{c}$, all sample sizes equal with $n$ total patients in the treatment group and also in the placebo group, and $\alpha=.05$.

If $n_{i j}=m_{i j}=n_{0}$ so that each patient has the same number of repeated episodes, and we make the assumption that the $x_{i j}$ and $y_{i j}$ have beta-binomial $(\rho)$ distributions, then

$$
\operatorname{Var}\left(x_{i j}\right)=n_{0} p_{t}\left(1-p_{t}\right)\left[1+\left(n_{0}-1\right) \rho\right] .
$$

The beta-binomial $(\rho)$ distribution arises from generating binomial counts with random success probability (See Section 3.1). The power approximation is then

$$
\Phi\left(\frac{\sqrt{n_{0}} \sqrt{n} \Delta}{\sqrt{\left[p_{t}\left(1-p_{t}\right)+p_{c}\left(1-p_{c}\right)\right]\left[1+\left(n_{0}-1\right) \rho\right]}}-1.96\right) .
$$

For $n=150$, and $\Delta=p_{t}-p_{c}=0.5-0.4=0.1$, we use $(3)$ to approximate the power for various $n_{0}$ when $\rho=0.3, \rho=0.5$ and $\rho=0.8$. The results are summarized in Figure 1.

From Figure 1, we can see that increasing the number of repeated measurements $\left(n_{0}\right)$ will increase the power of tests. However, the gain in power by increasing $n_{0}$ is small after $n_{0}=10$, with some dependence on $\rho$. Therefore, when planning a study, one could easily use (3) to jointly consider both the total sample size and the number of repeated measurements in order to minimize study cost and achieve the desired power. 


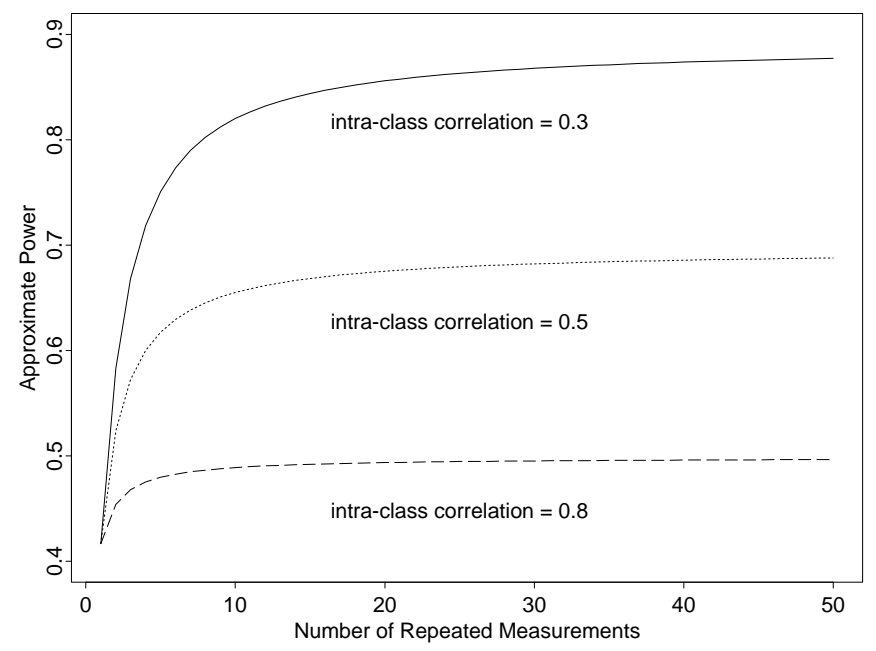

Figure 1: Power vs Number of Repeated Measurements

\section{Monte Carlo Study}

\subsection{Size Comparison}

A simulation study was conducted to assess the size of the two new test statistics, $T_{P}$ and $T_{U}$, and to compare them to the Mantel-Haenszel test statistic $T_{M H}, T_{L}$ from Liang (1985), and $T_{D D}$ from Donald and Donner (1987).

The total number of patients was fixed at 200, with 101 in the control group and 99 in the treatment group. The number of strata was chosen to be 5,15 , and 25 , and the number of patients in the control and treatment groups inside each stratum are summarized in Table 1. The numbers of repeated measurements $n_{i j}$ and $m_{i j}$ were fixed at 5 (columns $1,4,7$ ) and allowed to range from 5 to 10 (columns 2,5,8) and from 5 to 15 (columns 3,6,9). The control group success probability $p_{c i}$ ranged from 0.2 to 0.8 with increments $0.12(k=5), 0.04(\mathrm{k}=15)$, and $0.024(\mathrm{k}=25)$. The $x_{i j}$ and $y_{i j}$ were generated from the beta-binomial distribution with $\rho=0$ (binomial), $\rho=0.2$, and $\rho=0.8$. A total of 1000 simulated data sets were run for each combination of parameters. Since 1000 replicates were used, the standard error of estimates of both the size and the power in the simulation are smaller than $1 / \sqrt{4000}=0.0158$. SAS IML macros were used for all programming. 
The beta-binomial deviates $\left(x_{i j}\right)$ are generated in the following two steps. In the first step, beta deviates $\left(\pi_{i j}\right)$ are generated from a beta distribution $(\mathrm{a}, \mathrm{b})$ with $\mathrm{a}=p_{t i}(1-\rho) / \rho$ and $\mathrm{b}=$ $\left(1-p_{t i}\right)(1-\rho) / \rho$. In the second step, $x_{i j}$ are generated from a binomial distribution $\left(\pi_{i j}, n_{i j}\right)$. Then $x_{i j}$ has the beta-binomial density function with $\operatorname{Var}\left(x_{i j}\right)=n_{i j} p_{t i}\left(1-p_{t i}\right)\left[1+\left(n_{i j}-1\right) \rho\right]$, and $\rho$ is the intra-class correlation defined as the correlation between individual responses $x_{i j l}$ ( 0 or 1$)$ and $x_{i j l}(0$ or 1$)$ of the jth patient in the ith stratum. In fact, $\rho$ is the usual correlation

$$
\rho=\operatorname{Cov}\left(x_{i j l}, x_{i j l^{\prime}}\right) / \sqrt{\operatorname{Var}\left(x_{i j l}\right) \operatorname{Var}\left(x_{i j l^{\prime}}\right)} \text {. }
$$

From Table 2 we can see that the new tests hold their level very well across all situations, and perform very similarly. The Mantel-Haenszel test is of course far too liberal when $\rho>0$. Liang's $T_{L}$ tends to be too conservative when $\mathrm{k}=5$, but approximates the $5 \%$ level well when $\mathrm{k}=15$ and $\mathrm{k}=25$. Donald and Donner's $T_{D D}$ has erratic behavior for $k=15$ and $k=25$. 
Table 1. Number of patients in treatment groups at each center

\begin{tabular}{|c|c|c|c|c|c|c|}
\hline \multirow[b]{2}{*}{ stratum } & \multicolumn{2}{|c|}{$k=5$} & \multicolumn{2}{|c|}{$k=15$} & \multicolumn{2}{|c|}{$k=25$} \\
\hline & control & treatment & control & treatment & control & treatment \\
\hline 1 & 25 & 18 & 7 & 6 & 4 & 3 \\
\hline 2 & 16 & 15 & 10 & 10 & 3 & 4 \\
\hline 3 & 21 & 25 & 4 & 7 & 5 & 4 \\
\hline 4 & 17 & 23 & 8 & 6 & 5 & 3 \\
\hline 5 & 22 & 18 & 5 & 8 & 6 & 3 \\
\hline 6 & & & 7 & 6 & 4 & 4 \\
\hline 7 & & & 9 & 6 & 3 & 5 \\
\hline 8 & & & 5 & 8 & 4 & 6 \\
\hline 9 & & & 9 & 7 & 3 & 5 \\
\hline 10 & & & 8 & 8 & 3 & 4 \\
\hline 11 & & & 6 & 4 & 4 & 3 \\
\hline 12 & & & 5 & 5 & 3 & 3 \\
\hline 13 & & & 8 & 6 & 5 & 5 \\
\hline 14 & & & 6 & 6 & 4 & 3 \\
\hline 15 & & & 4 & 6 & 5 & 3 \\
\hline 16 & & & & & 3 & 5 \\
\hline 17 & & & & & 5 & 4 \\
\hline 18 & & & & & 4 & 6 \\
\hline 19 & & & & & 4 & 5 \\
\hline 20 & & & & & 5 & 3 \\
\hline 21 & & & & & 5 & 4 \\
\hline 22 & & & & & 4 & 3 \\
\hline 23 & & & & & 3 & 4 \\
\hline 24 & & & & & 3 & 3 \\
\hline 25 & & & & & 4 & 4 \\
\hline
\end{tabular}


Table 2. Estimates of size for nominal $\alpha=0.05$ tests for data from the beta-binomial $(\rho)$ distribution.

\begin{tabular}{clccccccccc}
\hline \hline \multirow{2}{*}{$\begin{array}{c}\text { Number of } \\
\text { strata }\end{array}$} & \multicolumn{4}{c}{$\rho=0.0$} & \multicolumn{3}{c}{$\rho=0.2$} & \multicolumn{3}{c}{$\rho=0.8$} \\
\cline { 2 - 10 }${ }_{\text {k }=5}, m_{i j}=$ & 5 & $5-10$ & $5-15$ & 5 & $5-10$ & $5-15$ & 5 & $5-10$ & $5-15$ \\
& $T_{M H}$ & .056 & .054 & .042 & .141 & .175 & .261 & .340 & .437 & .493 \\
& $T_{L}$ & .022 & .022 & .016 & .020 & .019 & .033 & .017 & .023 & .023 \\
& $T_{D D}$ & .046 & .038 & .032 & .051 & .050 & .066 & .044 & .049 & .043 \\
& $T_{P}$ & .057 & .052 & .039 & .050 & .050 & .064 & .041 & .047 & .036 \\
& $T_{U}$ & .058 & .058 & .040 & .051 & .051 & .067 & .045 & .048 & .040 \\
\hline \multirow{2}{*}{$\mathrm{k}=15$} & $T_{M H}$ & .047 & .065 & .032 & .154 & .215 & .238 & .325 & .432 & .482 \\
& $T_{L}$ & .049 & .051 & .037 & .045 & .051 & .039 & .040 & .038 & .038 \\
& $T_{D D}$ & .037 & .050 & .022 & .086 & .095 & .104 & .119 & .133 & .131 \\
& $T_{P}$ & .045 & .060 & .032 & .045 & .057 & .049 & .054 & .047 & .052 \\
& $T_{U}$ & .048 & .060 & .037 & .048 & .061 & .051 & .055 & .049 & .057 \\
\hline \multirow{2}{*}{$\mathrm{k}=25$} & $T_{M H}$ & .043 & .059 & .046 & .157 & .203 & .234 & .344 & .411 & .491 \\
& $T_{L}$ & .047 & .045 & .058 & .047 & .052 & .048 & .045 & .040 & .043 \\
& $T_{D D}$ & .024 & .029 & .024 & .049 & .057 & .064 & .081 & .091 & .093 \\
& $T_{P}$ & .044 & .057 & .044 & .049 & .055 & .053 & .047 & .050 & .054 \\
& $T_{U}$ & .042 & .059 & .043 & .047 & .053 & .052 & .049 & .048 & .053 \\
\hline \hline
\end{tabular}

\subsection{Power Comparison}

We use the same data structure as in Table 1 and Table 2, with data now generated under the alternative $H_{0}: \Psi=1.5$. Because the nominal size of $T_{D D}$ was not very stable in Table 2 , it was not included here. $T_{M H}$ results are only given when $\rho=0$. The results are summarized in Table 3.

Table 3 shows that when $\rho=0$, the power of $T_{P}$ and $T_{U}$ is almost equal to the power of the Mantel-Haenszel test. When $\mathrm{k}$ is small, the power of $T_{P}$ and $T_{U}$ is much better than $T_{L}$. With the increase in $\mathrm{k}$, the difference in power between $T_{L}$ and $T_{P}$ and $T_{U}$ decreases. However, when $\mathrm{k}=25$, the power of $T_{P}$ and $T_{U}$ is still considerably better than the power of $T_{L}$.

To check the approximate power expression (2), we calculated from (2) the powers .833 
$(\mathrm{k}=5), .844(\mathrm{k}=15)$, and $.839(\mathrm{k}=25)$ for the situation of column 1 of Table $3\left(n_{i j}=m_{i j}=5\right.$, $\rho=0.0)$. Similarly for column 5 of Table $3\left(n_{i j}=m_{i j}=5-10, \rho=0.2\right)$, we obtained powers of .638 $(\mathrm{k}=5), .645(\mathrm{k}=15)$, and $.636(\mathrm{k}=25)$. These and similar calculations convince us that (2)-(3) can be confidently used in planning studies with $T_{P}$ and $T_{U}$.

Table 3. Estimates of power when $\alpha=.05, \Psi=1.5$, and the data are beta-binomial $(\rho)$.

\begin{tabular}{|c|c|c|c|c|c|c|c|c|c|c|}
\hline \multirow{2}{*}{$\begin{array}{c}\text { Number of } \\
\text { strata }\end{array}$} & \multicolumn{4}{|c|}{$\rho=0.0$} & \multicolumn{3}{|c|}{$\rho=0.2$} & \multicolumn{3}{|c|}{$\rho=0.8$} \\
\hline & $n_{i j}, m_{i j}=$ & 5 & $5-10$ & 5-15 & 5 & $5-10$ & $5-15$ & 5 & $5-10$ & $5-15$ \\
\hline \multirow[t]{4}{*}{$k=5$} & $T_{M H}$ & .827 & .936 & .976 & & & & & & \\
\hline & $T_{L}$ & .378 & .465 & .582 & .199 & .202 & .205 & .083 & .104 & .090 \\
\hline & $T_{P}$ & .820 & .932 & .976 & .569 & .619 & .659 & .276 & .296 & .291 \\
\hline & $T_{U}$ & .825 & .936 & .977 & .570 & .624 & .660 & .280 & .304 & .291 \\
\hline \multirow[t]{4}{*}{$\mathrm{k}=15$} & $T_{M H}$ & .834 & .924 & .974 & & & & & & \\
\hline & $T_{L}$ & .758 & .876 & .937 & .521 & .553 & .585 & .267 & .237 & .237 \\
\hline & $T_{P}$ & .828 & .923 & .973 & .606 & .648 & .675 & .311 & .286 & .301 \\
\hline & $T_{U}$ & .836 & .926 & .973 & .603 & .647 & .684 & .317 & .301 & .271 \\
\hline \multirow[t]{4}{*}{$\mathrm{k}=25$} & $T_{M H}$ & .869 & .945 & .963 & & & & & & \\
\hline & $T_{L}$ & .821 & .917 & .953 & .551 & .587 & .580 & .246 & .280 & .270 \\
\hline & $T_{P}$ & .861 & .935 & .963 & .587 & .616 & .626 & .277 & .305 & .293 \\
\hline & $T_{U}$ & .865 & .941 & .963 & .596 & .618 & .630 & .281 & .309 & .300 \\
\hline
\end{tabular}

\section{Confidence Intervals for the Common Odds Ratio $\Psi$}

\subsection{Two Proposed Confidence Intervals}

To derive confidence intervals for the common odds ratio $\Psi$, we need to generalize $T_{L}$ and $T_{U}$ to test $H_{0}: \Psi=\Psi_{0}$. Then we will invert the resulting tests to get confidence intervals for $\Psi$.

The generalization of Liang's statistic $T_{L}$ is

$$
T_{L, \Psi_{0}}=\frac{\left(\sum_{i=1}^{k} u_{i}\right)^{2}}{\sum_{i=1}^{k} u_{i}^{2}},
$$

where 


$$
u_{i}=\frac{x_{i .}\left(m_{i .}-y_{i .}\right)}{N_{i}}-\Psi_{0} \frac{\left(n_{i .}-x_{i .}\right) y_{i .}}{N_{i}}
$$

$T_{L, \Psi_{0}}$ converges to a $\chi_{1}^{2}$ distribution as $k \rightarrow \infty$ under $H_{0}: \Psi=\Psi_{0}$.

The generalization of $T_{U}$ is

$$
T_{U, \Psi_{0}}=\frac{\left(\sum_{i=1}^{k} u_{i}\right)^{2}}{V_{U, \Psi_{0}}}
$$

where

$$
\begin{gathered}
V_{U, \Psi_{0}}=\sum_{i=1}^{k} N_{i}^{-2}\left\{m_{i}^{2} A_{i}+\Psi_{0}^{2} n_{i .}^{2} B_{i}+\Psi_{0}^{2} n_{i .}^{2} B_{i}+\left(1-\Psi_{0}\right)^{2}\left(A_{i} B_{i}+x_{i .}^{2} B_{i}+A_{i} y_{i .}^{2}\right)\right. \\
\left.-\left(1-\Psi_{0}\right) m_{i .} A_{i} y_{i .}+\Psi_{0}\left(1-\Psi_{0}\right) n_{i .} B_{i} x_{i .}\right\}
\end{gathered}
$$

and

$$
A_{i}=\frac{1}{\delta_{t i}} \sum_{j=1}^{n_{i}} \frac{\left(x_{i j}-n_{i j} \hat{p}_{t i}\right)^{2}}{\left(1-2 n_{i j} / n_{i .}\right)}, \quad B_{i}=\frac{1}{\delta_{c i}} \sum_{j=1}^{m_{i}} \frac{\left(y_{i j}-m_{i j} \hat{p}_{c i}\right)^{2}}{\left(1-2 m_{i j} / m_{i .}\right)}
$$

We have not generalized $T_{P}$ because the pooled proportion estimators $\hat{p}_{i}$ do not make sense unless $\Psi_{0}=1$. Similar to the results for $V_{U}, V_{U, \Psi_{0}}$ is unbiased for $E \sum_{i=1}^{k} u_{i}^{2}$ under $H_{0}: \Psi=\Psi_{0}$, and $T_{U, \Psi_{0}}$ converges to a $\chi_{1}^{2}$ distribution for either $k \rightarrow \infty$ or for the number of patients per strata $\rightarrow \infty$.

Thus our confidence intervals are the sets $C_{L}=\left\{\Psi: T_{L, \Psi}<\chi_{1}^{2}(1-\alpha)\right\}$ and $C_{U}=\{\Psi$ : $\left.T_{U, \Psi}<\chi_{1}^{2}(1-\alpha)\right\}$, where $\chi_{1}^{2}(1-\alpha)$ is the $(1-\alpha)$ quantile of the $\chi_{1}^{2}$ distribution. Some algebra reveals that each of these sets has the form

$$
\left\{\Psi: \frac{a_{1} \Psi^{2}+b_{1} \Psi+c_{1}}{a_{2} \Psi^{2}+b_{2} \Psi+c_{2}}<\chi_{1}^{2}(1-\alpha)\right\}
$$

where the constants $a_{1}, b_{1}, c_{1}, a_{2}, b_{2}, c_{2}$ are given in the Appendix. If $a_{1}-a_{2} \chi_{1}^{2}(1-\alpha)>0$, then the sets are indeed intervals. In practice $C_{L}$ almost always gives closed intervals as long as $k \geq 15$, and $C_{U}$ generally gives closed intervals (only 17 out of 27000 data sets used in constructing Table 4 failed to result in closed intervals). 


\subsection{Monte Carlo Study}

We use the same data structure as in Tables 1-3 with true odds ratio equal to 1.5. The results are summarized in Table 4.

The simulation results show that both $C_{L}$ and $C_{U}$ approximate the nominal $95 \%$ coverage probability well. When $\mathrm{k}=5, C_{U}$ has much smaller shorter length than $C_{L}$. Note also that about $10 \%$ of the $C_{L}$ sets were not closed intervals for $k=5$ and thus were not included in the Table 3 results.

When k=15, both $C_{L}$ and $C_{U}$ have almost the same length. When k=25, $C_{L}$ has a shorter length than $C_{U}$. Keep in mind that for $k=25$ there are only 8 patients on average in each of the strata. Thus, in contrast to the testing results where $T_{P}$ and $T_{U}$ could be recommended in all situations studied, here it appears that the confidence interval from Liang's method can be preferable in the many small strata situation.

Table 4. Coverage and length for $95 \%$ confidence intervals for data from the beta-binomial $(\rho)$ distribution with $\Psi=1.5$.

\begin{tabular}{|c|c|c|c|c|c|c|c|c|c|c|c|}
\hline \multirow{2}{*}{$\begin{array}{c}\text { Number of } \\
\text { strata }\end{array}$} & & & \multicolumn{3}{|c|}{$\rho=0.0$} & \multicolumn{3}{|c|}{$\rho=0.2$} & \multicolumn{3}{|c|}{$\rho=0.8$} \\
\hline & & $n_{i j}, m_{i j}=$ & 5 & $5-10$ & $5-15$ & 5 & $5-10$ & $5-15$ & 5 & $5-10$ & $5-15$ \\
\hline \multirow[t]{4}{*}{$\mathrm{k}=5$} & $C_{L}$ & Coverage & .977 & .977 & .977 & .984 & .980 & .975 & .964 & .976 & .981 \\
\hline & & Mean Length & 1.93 & 1.48 & 1.25 & 5.15 & 2.88 & 3.95 & 11.6 & 12.9 & 10.6 \\
\hline & $C_{U}$ & Coverage & .969 & .964 & .968 & .977 & .968 & .961 & .947 & .957 & .951 \\
\hline & & Mean Length & 0.95 & 0.77 & 0.69 & 1.42 & 1.29 & 1.26 & 3.32 & 4.34 & 3.80 \\
\hline \multirow[t]{4}{*}{$k=15$} & $C_{L}$ & Coverage & .944 & .955 & .952 & .959 & .951 & .959 & .936 & .947 & .964 \\
\hline & & Mean Length & 0.94 & 0.80 & 0.69 & 1.32 & 1.26 & 1.21 & 2.31 & 2.39 & 2.50 \\
\hline & $C_{U}$ & Coverage & .948 & .962 & .956 & .963 & .957 & .959 & .955 & .956 & .963 \\
\hline & & Mean Length & 0.94 & 0.78 & 0.68 & 1.35 & 1.29 & 1.23 & 3.01 & 3.73 & 3.40 \\
\hline \multirow[t]{4}{*}{$k=25$} & $C_{L}$ & Coverage & .957 & .949 & .956 & .959 & .962 & .954 & .961 & .959 & .960 \\
\hline & & Mean Length & 0.90 & 0.77 & 0.68 & 1.24 & 1.16 & 1.17 & 2.17 & 2.25 & 2.29 \\
\hline & $C_{U}$ & Coverage & .962 & .962 & .959 & .969 & .969 & .959 & .964 & .962 & .964 \\
\hline & & Mean Length & 0.94 & 0.78 & 0.71 & 1.36 & 1.27 & 1.26 & 3.16 & 3.51 & 4.72 \\
\hline
\end{tabular}




\section{$5 \quad$ Examples}

For illustrative purpose we have carried out the analyses for both examples using the standard Mantel-Haenszel statistic $T_{M H}$, Liang's statistic $T_{L}$, and our new statistics $T_{P}$ and $T_{L}$.

For Example 1, the standard Mantel-Haenszel statistic $T_{M H}=53.93$. Liang's statistic $T_{L}=7.84$ with p-value .0051 , whereas $T_{P}=20.26$ with $\mathrm{p}$-value $<.0001$, and $T_{U}=22.83$ with p-value $<.0001$. For Example 2, since the real data could not be released, we generated the responses $x_{i j}$ and $y_{i j}$ using the same method as discussed in the Monte Carlo Study Section with $\rho=.3$ and average $p_{t i}=.5$ and $p_{c i}=.4$ with the odds ratio $\Psi$ held constant at $\Psi=1.5$. These values are estimates from the actual data. But the number of patients in each center is taken from the real study. The results are the average values from ten runs with different seeds. The average of the standard Mantel-Haenszel statistic is $\bar{T}_{M H}=52.52$, the average of Liang's statistic is $\bar{T}_{L}=6.44$ with average p-value .0202 , while $\bar{T}_{P}=11.65$ with average p-value .0084 , and $\bar{T}_{U}=12.00$ with average p-value .0086 .

From the results, we can see that $T_{M H}$ is overly optimistic due to the correlation within individual patients, and our new test statistics $T_{P}$ and $T_{L}$ are more powerful than $T_{L}$ which agrees with the simulation results in Table 3 .

Note that we can still analyze the data in Example 1 by using $T_{M H}$ on data at each visit. But we cannot apply this approach to Example 2 since the time of onset of heartburn symptoms is different for each patient, and the number of episodes is different, too. Therefore this approach is not always applicable. More important, an overall conclusion as to whether the new drug constitutes an "improvement" over the placebo for the entire study is often interesting in addition to the separate result for each occasion.

For Example 1, the $95 \%$ confidence interval for the odds ratio $\Psi$ from inverting Liang's statistic is $(1.66,6.78)$, while the interval obtained from $T_{U}$ is $(1.78,12.91)$. For Example 2, the average $95 \%$ confidence interval for the odds ratio $\Psi$ from inverting Liang's statistic is (1.15, $1.95)$, while the interval obtained from $T_{U}$ is $(1.18,1.95)$. Recall that Table 4 shows an advantage for Liang's intervals in terms of average length. 


\section{Concluding Remarks}

We have introduced two new test statistics for correlated binary data which perform well for a wide range of strata sizes and number of strata. They were motivated by randomized clinical trials, but the results can also be applied to cohort and case-control studies.

The statistic $T_{P}$ which uses pooled estimators in the variance estimate is our preference. It is the same as Liang's (1985) $T_{L}$ in the case of matched pairs and also reduces to a SAS CMH statistic in the case where the number of episodes per patient is held constant. The statistic $T_{U}$ which uses unpooled strata proportion estimators in its variance estimate performed very similar to $T_{P}$ in the Monte Carlo studies. Both $T_{P}$ and $T_{L}$ have power advantages over $T_{L}$, especially for a small number of strata. In addition, the approximate power calculations of Section 2.3 are easy to use in the practical design of a clinical trial.

For confidence intervals for $\Psi$, the interval based on $T_{U}$ is much better than that based on $T_{L}$ when the number of strata is small. The situation is reversed when the number of strata is large with very few patients per stratum.

We might add that all the methods discussed in this paper are such that the binary outcomes are equally weighted within strata. Some researchers would prefer that patients be equally weighted instead. One simple approach is to use ANOVA methods on the variables $\hat{p}_{x i j}=x_{i j} / n_{i j}$ and $\hat{p}_{y i j}=y_{i j} / m_{i j}$. We implemented this approach using the F statistic for treatment with SAS Type III sums of squares in a model with strata, treatment, and strata by treatment interaction (see Searle, 1987). The simulation results are not given because of limited space, but they were very similar to those obtained by $T_{P}$ and $T_{U}$ in Tables 2 and 3 except that the power was slightly smaller on average.

\section{Acknowledgement}

We would like to thank El Giefer whose broad experience with analyzing clinical trial data motivated the results of this paper. 


\section{Appendix}

Table A1. Distribution of successful response to drug and placebo from the psoriasis clinical trial.

\begin{tabular}{|c|c|c|c|c|c|c|}
\hline \multirow[b]{2}{*}{ Center } & \multirow[b]{2}{*}{ Treatment } & \multirow{2}{*}{$\begin{array}{c}\text { Number of } \\
\text { patients }\end{array}$} & \multicolumn{2}{|c|}{ Responses } & \multirow[b]{2}{*}{ Total } & \multirow{2}{*}{$\begin{array}{c}\text { Proportion } \\
\text { success }\end{array}$} \\
\hline & & & Successes & Failures & & \\
\hline \multirow{3}{*}{1} & & $\underline{n_{i}, m_{i}}$ & $\underline{x_{i .}, y_{i}}$ & $\begin{array}{l}n_{i .}-x_{i} \\
m_{i .}-y_{i .}\end{array}$ & $\underline{n_{i .}, m_{i}}$ & \\
\hline & Drug & 12 & $\overline{24}$ & 13 & 37 & 0.649 \\
\hline & Placebo & 12 & 17 & 12 & 29 & 0.586 \\
\hline \multirow[t]{2}{*}{2} & Drug & 6 & 17 & 3 & 20 & 0.850 \\
\hline & Placebo & 5 & 13 & 7 & 20 & 0.650 \\
\hline \multirow[t]{2}{*}{3} & Drug & 8 & 20 & 0 & 20 & 1.000 \\
\hline & Placebo & 8 & 22 & 7 & 29 & 0.759 \\
\hline \multirow[t]{2}{*}{4} & Drug & 10 & 38 & 2 & 40 & 0.950 \\
\hline & Placebo & 10 & 21 & 18 & 39 & 0.538 \\
\hline \multirow[t]{2}{*}{5} & Drug & 12 & 38 & 4 & 42 & 0.905 \\
\hline & Placebo & 12 & 25 & 20 & 45 & 0.556 \\
\hline \multirow[t]{2}{*}{6} & Drug & 4 & 12 & 1 & 13 & 0.923 \\
\hline & Placebo & 5 & 8 & 11 & 19 & 0.421 \\
\hline \multirow[t]{2}{*}{7} & Drug & 8 & 16 & 16 & 32 & 0.500 \\
\hline & Placebo & 8 & 13 & 6 & 19 & 0.684 \\
\hline \multirow[t]{2}{*}{8} & Drug & 8 & 29 & 0 & 29 & 1.000 \\
\hline & Placebo & 8 & 28 & 4 & 32 & 0.875 \\
\hline \multirow[t]{2}{*}{9} & Drug & 8 & 27 & 4 & 31 & 0.871 \\
\hline & Placebo & 8 & 10 & 19 & 29 & 0.345 \\
\hline \multirow[t]{2}{*}{10} & Drug & 12 & 40 & 5 & 45 & 0.889 \\
\hline & Placebo & 11 & 31 & 11 & 42 & 0.738 \\
\hline \multirow[t]{2}{*}{11} & Drug & 11 & 38 & 4 & 42 & 0.905 \\
\hline & Placebo & 11 & 35 & 6 & 41 & 0.854 \\
\hline \multirow[t]{2}{*}{12} & Drug & 8 & 25 & 7 & 32 & 0.781 \\
\hline & Placebo & 10 & 28 & 11 & 39 & 0.718 \\
\hline \multirow[t]{2}{*}{13} & Drug & 8 & 23 & 7 & 30 & 0.767 \\
\hline & Placebo & 9 & 29 & 5 & 34 & 0.853 \\
\hline \multirow[t]{2}{*}{14} & Drug & 12 & 39 & 5 & 44 & 0.886 \\
\hline & Placebo & 11 & 33 & 11 & 44 & 0.750 \\
\hline \multirow[t]{2}{*}{15} & Drug & 8 & 28 & 3 & 31 & 0.903 \\
\hline & Placebo & 7 & 23 & 5 & 28 & 0.821 \\
\hline \multirow[t]{2}{*}{16} & Drug & 8 & 32 & 0 & 32 & 1.000 \\
\hline & Placebo & 8 & 17 & 15 & 32 & 0.531 \\
\hline
\end{tabular}


Table A2. Response from all 11 patients in Center 2 from the psoriasis clinical trial (Patient 11 dropped out).

\begin{tabular}{|c|c|c|c|c|}
\hline Patients & Treatment & $\begin{array}{l}\text { Number of } \\
\text { successes }\end{array}$ & $\begin{array}{c}\text { Number of } \\
\text { failures }\end{array}$ & $\begin{array}{c}\text { Total number } \\
\text { of visits }\end{array}$ \\
\hline 1 & Placebo & $y_{21}=1$ & 3 & $m_{21}=4$ \\
\hline 2 & Placebo & $y_{22}=4$ & 0 & $m_{22}=4$ \\
\hline 3 & Placebo & $y_{23}=4$ & 0 & $m_{23}=4$ \\
\hline 4 & Placebo & $y_{24}=4$ & 0 & $m_{24}=4$ \\
\hline 5 & Placebo & $y_{25}=0$ & 4 & $m_{25}=4$ \\
\hline 6 & Drug & $x_{21}=4$ & 0 & $n_{21}=4$ \\
\hline 7 & Drug & $x_{22}=3$ & 1 & $n_{22}=4$ \\
\hline 8 & Drug & $x_{23}=4$ & 0 & $n_{23}=4$ \\
\hline 9 & Drug & $x_{24}=4$ & 0 & $n_{24}=4$ \\
\hline 10 & Drug & $x_{25}=2$ & 2 & $n_{25}=4$ \\
\hline 11 & Drug & $x_{26}=0$ & 0 & $n_{26}=0$ \\
\hline
\end{tabular}


Table A3. Data structure for Example 2 based on centers.

\begin{tabular}{|c|c|c|c|c|c|c|}
\hline \multirow[b]{2}{*}{ Center } & \multirow[b]{2}{*}{ Treatment } & \multirow{2}{*}{$\begin{array}{c}\text { Number of } \\
\text { patients }\end{array}$} & \multicolumn{2}{|c|}{ 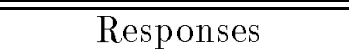 } & \multirow[b]{2}{*}{ Total } & \multirow{2}{*}{$\begin{array}{c}\text { Proportion } \\
\text { success }\end{array}$} \\
\hline & & & Successes & Failures & & \\
\hline \multirow{3}{*}{1} & & $n_{i}, m_{i}$ & $x_{i}, y_{i}$ & $\begin{array}{l}n_{i .}-x_{i} \\
m_{i}-y_{i}\end{array}$ & $n_{i} . m_{i}$ & \\
\hline & Drug & 6 & 21 & 34 & 55 & 0.382 \\
\hline & Placebo & 6 & 28 & 37 & 65 & 0.431 \\
\hline \multirow[t]{2}{*}{2} & Drug & 16 & 93 & 76 & 169 & 0.550 \\
\hline & Placebo & 16 & 72 & 81 & 153 & 0.471 \\
\hline \multirow[t]{2}{*}{3} & Drug & 12 & 80 & 63 & 143 & 0.559 \\
\hline & Placebo & 15 & 43 & 109 & 152 & 0.283 \\
\hline$\vdots$ & $\vdots$ & $\vdots$ & $\vdots$ & $\vdots$ & $\vdots$ & $\vdots$ \\
\hline \multirow[t]{2}{*}{17} & Drug & 15 & 66 & 93 & 159 & 0.415 \\
\hline & Placebo & 16 & 38 & 112 & 150 & 0.253 \\
\hline
\end{tabular}

Table A4. Data structure for Example 1 based on patients (Center 1).

\begin{tabular}{clccc}
\hline \hline Patients & Treatment & $\begin{array}{c}\text { Number of } \\
\text { successes }\end{array}$ & $\begin{array}{c}\text { Number of } \\
\text { failures }\end{array}$ & $\begin{array}{c}\text { Total number } \\
\text { of episodes }\end{array}$ \\
\hline & Placebo & $y_{11}=2$ & 17 & $m_{11}=19$ \\
1 & Placebo & $y_{12}=6$ & 5 & $m_{12}=11$ \\
3 & Placebo & $y_{13}=9$ & 7 & $m_{13}=16$ \\
4 & Placebo & $y_{14}=5$ & 0 & $m_{14}=5$ \\
5 & Placebo & $y_{15}=6$ & 3 & $m_{15}=9$ \\
6 & Placebo & $y_{16}=0$ & 5 & $m_{16}=5$ \\
& & & & \\
7 & Drug & $x_{11}=4$ & 7 & $n_{11}=11$ \\
8 & Drug & $x_{12}=0$ & 6 & $n_{12}=6$ \\
9 & Drug & $x_{13}=5$ & 5 & $n_{13}=10$ \\
10 & Drug & $x_{14}=9$ & 8 & $n_{14}=17$ \\
11 & Drug & $x_{15}=1$ & 2 & $n_{15}=3$ \\
12 & Drug & $x_{16}=2$ & 6 & $n_{16}=8$ \\
\hline \hline
\end{tabular}

Note: since the real data could not be released, the responses were generated using the estimated parameters from the actual data. But the number of patients in each center is taken from the real study. 
Theorem 3. If the $x_{i j}$ and $y_{i j}$ are all independent with means $E\left(x_{i j}\right)=n_{i j} p_{i}$ and $E\left(y_{i j}\right)=$ $m_{i j} p_{i}$, the sample sizes $n_{i j}$ and $m_{i j}$ are bounded by $N_{0}<\infty$, the variances $\operatorname{Var}\left(x_{i j}\right)$ and $\operatorname{Var}\left(y_{i j}\right)$ are bounded below by $c_{0}>0$, and $\sum_{i=1}^{k}\left\{\left(1-\lambda_{i}\right)^{2} n_{i}+\lambda_{i}^{2} m_{i}\right\} \rightarrow \infty$, then

$$
\frac{\left\{\sum_{i=1}^{k}\left(x_{i .}-n_{i .} t_{i .} / N_{i}\right)\right\}^{2}}{\sum_{i=1}^{k}\left\{\left(1-\lambda_{i}\right)^{2} \sum_{j=1}^{n_{i}}\left(x_{i j}-n_{i j} p_{i}\right)^{2}+\lambda_{i}^{2} \sum_{j=1}^{m_{i}}\left(y_{i j}-m_{i j} p_{i}\right)^{2}\right\}} \stackrel{d}{\rightarrow} \chi_{1}^{2} .
$$

To get the null asymptotic chi-squared result for $T_{P}$ and $T_{U}$, we need only to show that the ratios of $V_{P}$ and $V_{U}$ to the denominator in Theorem 3 converge to 1 in probability. This results in the following theorem.

Theorem 4. Assume that either i) $k \rightarrow \infty$ with $1 \leq n_{i} \leq c_{1}<\infty$ and $1 \leq m_{i} \leq c_{1}<\infty$ or ii) $k$ remains bounded with $\min \left(n_{i}, m_{i}\right) \rightarrow \infty$ for $i=1, \ldots, k$. Then under the assumptions of Theorems 1 and $3, T_{P} \stackrel{d}{\rightarrow} \chi_{1}^{2}$, and under the assumptions of Theorems 2 and $3, T_{U} \stackrel{d}{\rightarrow} \chi_{1}^{2}$.

Confidence interval details. As mentioned in Section 4.1, both $C_{L}$ and $C_{U}$ are obtained by solving

$$
\left.\frac{a_{1} \Psi^{2}+b_{1} \Psi+c_{1}}{a_{2} \Psi^{2}+b_{2} \Psi+c_{2}}<\chi_{1}^{2}(1-\alpha)\right\}
$$

For $C_{L}$ the constants are

$$
\begin{aligned}
& a_{1}=\left\{\sum_{i=1}^{k} \frac{\left(n_{i .}-x_{i .}\right) y_{i .}}{N_{i}}\right\}^{2}, b_{1}=-2 \sum_{i=1}^{k} \frac{\left(n_{i .}-x_{i .}\right) y_{i .}}{N_{i}} \sum_{i=1}^{k} \frac{x_{i .}\left(m_{i .}-y_{i .}\right)}{N_{i}}, c_{1}=\left\{\sum_{i=1}^{k} \frac{\left(m_{i .}-y_{i .}\right) x_{i .}}{N_{i}}\right\}^{2}, \\
& a_{2}=\sum_{i=1}^{k}\left\{\frac{\left(n_{i .}-x_{i .}\right) y_{i .}}{N_{i}}\right\}^{2}, b_{2}=-2 \sum_{i=1}^{k} \frac{\left(n_{i .}-x_{i .}\right) y_{i .} x_{i .}\left(m_{i .}-y_{i .}\right)}{N_{i}{ }^{2}}, c_{2}=\sum_{i=1}^{k}\left\{\frac{\left(m_{i .}-y_{i .}\right) x_{i .}}{N_{i}}\right\}^{2} .
\end{aligned}
$$

For $C_{U}, a_{1}, b_{1}, c_{1}$ are the same, but

$$
\begin{aligned}
& a_{2}=\sum_{i=1}^{k}\left(n_{i .} / N_{i}\right)^{2} B_{i}+\sum_{i=1}^{k}\left(1 / N_{i}\right)^{2}\left(A_{i} B_{i}+x_{i .}^{2} B_{i}+A_{i} y_{i .}^{2}\right)-\sum_{i=1}^{k}\left(n_{i .} /\left(N_{i}\right)^{2} B_{i} x_{i .},\right. \\
& b_{2}=-2 \sum_{i=1}^{k}\left(1 / N_{i}\right)^{2}\left(A_{i} B_{i}+x_{i .}^{2} B_{i}+A_{i} y_{i .}^{2}\right)-\sum_{i=1}^{k}\left(n_{i .} / N_{i}^{2}\right) B_{i} x_{i .}, \\
& c_{2}=\sum_{i=1}^{k}\left(m_{i .} / N_{i}\right)^{2} A_{i}+\sum_{i=1}^{k}\left(1 / N_{i}\right)^{2}\left(A_{i} B_{i}+x_{i .}^{2} B_{i}+A_{i} y_{i .}^{2}\right)-\sum_{i=1}^{k}\left(m_{i .} / N_{i}^{2}\right) A_{i} y_{i .},
\end{aligned}
$$


where

$$
A_{i}=\frac{1}{\delta_{t i}} \sum_{j=1}^{n_{i}} \frac{\left(x_{i j}-n_{i j} \hat{p}_{t i}\right)^{2}}{\left(1-2 n_{i j} / n_{i .}\right)}, \quad B_{i}=\frac{1}{\delta_{c i}} \sum_{j=1}^{m_{i}} \frac{\left(y_{i j}-m_{i j} \hat{p}_{c i}\right)^{2}}{\left(1-2 m_{i j} / m_{i .}\right)}
$$

\section{REFERENCES}

Breslow, N.E. (1981). Odds ratio estimators when the data are sparse. Biometrika 68, 73-84.

Cochran, W. G. (1954). Some methods of strengthening the common $\chi^{2}$ tests. Biometrics 10 , 417-451.

Donald, A., and Donner, A. (1987). Adjustments to the Mantel-Haenszel chi-square statistics and odds ratio variance estimator when the data are clustered. Statistics in Medicine 6, 491-499.

Donald, A., and Donner, A. (1990). A simulation study of the analysis of sets of $2 \times 2$ contingency tables under cluster sampling: estimation of a common odds ratio. Journal of the American Statistical Association 85, 537-543.

Fitzmaurice, G.M., Laird, N.M. and Rotnitzky A.G. (1993), Regression models for discrete longitudinal responses. Statistical Science 8, 284-309.

Liang, K.Y. (1985). Odds ratio inference with dependent data. Biometrika 72, 678-682.

Liang, K.Y. and Zeger, S.L. (1986). Longitudinal data analysis using generalized linear models. Biometrika 73, 13-22.

Liang, K.Y. and Zeger, S.L. (1995). Inference based on estimating functions in the presence of nuisance parameters. Statistical Science 10, 158-199.

Rotnitsky, A. and Jewell, N.P. (1990). Hypothesis testing of regression parameters in semiparametric generalized linear models for cluster correlated data. Biometrika 77, 485-497.

Searle, S. R. (1987). Linear Models for Unbalanced Data. New York: John Wiley \& Sons, Inc.

Wittes, J. and Wallenstein, S. (1987). The power of the Mantel-Haenszel test. Journal of the American Statistical Association 82, 1104-1109. 
Zhang, J. and Boos, D.D. (1995). Mantel-Haenszel test statistics for correlated binary data. Institute of Statistics Mimeo Series No. 2274. 